\title{
Electromagnetic corrections to light hadron masses
}

\section{A. Portelli ${ }^{* b}$, S. Dürr ${ }^{a, d}$, Z. Fodor ${ }^{a, c, d}$, J. Frison ${ }^{b}$, C. Hölbling ${ }^{a}$, S.D. Katz ${ }^{a, c}$,

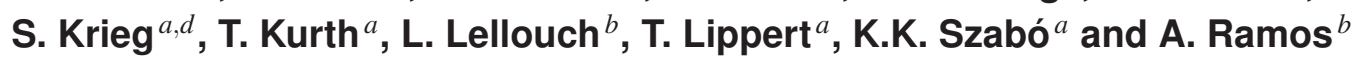 (Budapest-Marseille-Wuppertal Collaboration)}

${ }^{a}$ Bergische Universität Wuppertal, Gaußstr. 20, D-42097 Wuppertal, Germany

${ }^{b}$ Centre de Physique Théorique, Luminy, Case 907, F-13288 Marseille cedex 9, France ${ }^{\dagger}$

${ }^{c}$ Institute for Theoretical Physics, Eötvös University, H-1117 Budapest, Hungary

${ }^{d}$ Jülich Supercomputing Centre, Forschungszentrum Jülich, D-52425 Jülich, Germany

E-mail : portelliecpt.univ-mrs.fr

\begin{abstract}
At the precision reached in current lattice QCD calculations, electromagnetic effects are becoming numerically relevant. We will present preliminary results for electromagnetic corrections to light hadron masses, based on simulations in which a $\mathrm{U}(1)$ degree of freedom is superimposed on $N_{f}=2+1$ QCD configurations from the BMW collaboration.
\end{abstract}

The XXVIII International Symposium on Lattice Field Theory

June 14-19,2010

Villasimius, Sardinia Italy

\footnotetext{
* Speaker.

${ }^{\dagger}$ CPT is research unit UMR 6207 of the CNRS and of the universities Aix-Marseille II, Aix-Marseille I and Sud Toulon-Var, and is affiliated with the FRUMAM
} 


\section{Motivation}

Isospin is a near symmetry of the hadron spectrum because the strong interaction only distinguishes quark flavors through their masses and the mass difference between up and down quarks is small. This symmetry is broken by :

(i) the mass difference $m_{u}-m_{d}$ (mass isospin breaking)

(ii) the difference in the charge of the $u$ and the $d$ quark (electromagnetic isospin breaking)

\begin{tabular}{|l|c|c|}
\cline { 2 - 3 } \multicolumn{1}{c|}{} & $u$ & $d$ \\
\hline Mass (MeV) [1] & $2.49_{-0.79}^{+0.81}$ & $5.05_{-0.95}^{+0.75}$ \\
Charge & $\frac{2}{3} e$ & $-\frac{1}{3} e$ \\
\hline
\end{tabular}

These effects are expected to be at the percent level. The size of mass breaking is the mass difference $m_{u}-m_{d}$ relatively to a typical QCD scale $\Lambda_{\mathrm{QCD}}$ and the order of electromagnetic breaking is the fine structure constant at zero momentum $\alpha=\frac{e^{2}}{4 \pi} \simeq \frac{1}{137}$.

These effects imply mass splittings inside isospin multiplets. Although these effects are small, they have important consequences. For example, the fact that the neutron is heavier than the proton guarantees the stability of matter. Another interesting isospin breaking quantity is the absolute correction to Dashen's theorem:

$$
\Delta_{A} D=\Delta_{\mathrm{EM}} M_{K}^{2}-\Delta_{\mathrm{EM}} M_{\pi}^{2}
$$

where :

$$
\Delta_{\mathrm{EM}} M_{P}^{2}=\left(M_{P^{+}}^{2}-M_{P^{0}}^{2}\right)_{m_{u}=m_{d}}
$$

is the electromagnetic squared mass splitting of the isospin multiplet $P$. One can also consider the dimensionless relative correction to Dashen's theorem :

$$
\Delta_{R} D=\frac{\Delta_{\mathrm{EM}} M_{K}^{2}}{\Delta_{\mathrm{EM}} M_{\pi}^{2}}-1
$$

Dashen has shown in [2] than $\Delta_{A} D=0$ in the $\mathrm{SU}(3)$ chiral limit and that the leading corrections are $\mathrm{O}\left(\alpha m_{s}, \alpha^{2}\right)$. The quantity $\Delta_{A} D$ is interesting because it is very sensitive to the up and down quark masses.

An overview of the results for the violations to Dashen's theorem corrections is presented in Table 1. These results are interesting for two reasons : on one hand, agreement between different calculations is poor and on the other all these numbers predict rather large corrections. We will investigate corrections to Dashen's theorem using a QCD+QED analysis on BMW collaboration QCD ensembles. A first step in this direction is to formulate electromagnetism on the lattice. 


\begin{tabular}{|c|c|c|}
\hline & $\Delta_{A} D\left(\mathrm{MeV}^{2}\right)$ & $\Delta_{R} D$ \\
\hline \multirow{6}{*}{$\begin{array}{l}\text { do } \\
0 \\
0 \\
0 \\
0 \\
0 \\
0 \\
\frac{0}{0} \\
\frac{0}{2}\end{array}$} & 1230 & 0.80 \\
\hline & $1300 \pm 400$ & $1.02 \pm 0.30$ \\
\hline & 360 & 0.26 \\
\hline & $1060 \pm 320$ & $0.84 \pm 0.24$ \\
\hline & 1080 & 0.68 \\
\hline & 1070 & 0.85 \\
\hline \multirow{3}{*}{ 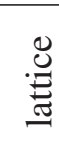 } & 526 & 0.39 \\
\hline & $340 \pm 92$ & $0.30 \pm 0.08$ \\
\hline & $1250 \pm 550$ & N/A \\
\hline
\end{tabular}

Table 1: Results for the violations to Dashen's theorem. Bold numbers are the results given by the authors, the others are deduced from information given in the corresponding paper.

\section{Electromagnetism on the lattice}

We impose periodic boundary condition in a finite volume, i.e. we will define Maxwell's theory on a 4-torus $\mathbb{T}^{4}$. In this context, the Maxwell-Gauss equations :

$$
\sum_{\mu=0}^{3} \partial_{\mu} F_{\mu v}=j_{v} \quad \text { with } \quad F_{\mu v}=\partial_{\mu} A_{v}-\partial_{v} A_{\mu}
$$

imposes global electric neutrality :

$$
Q_{\text {total }}=\int_{\mathbb{T}^{3}} \mathrm{~d}^{3} \mathbf{x} j_{0}(x)=\sum_{k=1}^{3} \int_{\mathbb{T}^{3}} \mathrm{~d}^{3} \mathbf{x} \partial_{k} F_{k 0}(x)=0
$$

One way to circumvent this global neutrality is to add a finite-volume, constant background current density as a multiplier to the Lagrangian of the theory :

$$
\mathscr{L}[A](c)=\frac{1}{4} \sum_{\mu, v=0}^{3} F_{\mu \nu} F_{\mu \nu}+\frac{1}{V} \sum_{\mu=0}^{3} L_{\mu} c_{\mu} A_{\mu}
$$

where $L_{\mu}$ is the length of direction $\mu$ in $\mathbb{T}^{4}$ and $V=L_{0} L_{1} L_{2} L_{3}$ is the 4-volume of $\mathbb{T}^{4}$. The MaxwellGauss equations become :

$$
\sum_{\mu=0}^{3} \partial_{\mu} F_{\mu \nu}=j_{v}-\frac{1}{V} L_{v} c_{v}
$$

Minimizing the action with respect to the Lagrange multiplier $c$ yields a new Euler-Lagrange equation :

$$
\hat{A}_{\mu}(0)=\int_{\mathbb{T}^{4}} \mathrm{~d}^{4} x A_{\mu}(x)=0
$$

where the hat symbol denotes Fourier transform.

We formulate now electromagnetism on the lattice in its non-compact form (following [9]). This is done by using forward finite differences in the Lagrangian (2.3) :

$$
\partial_{\mu} f(x)=f(x+\hat{\mu})-f(x)
$$


This formulation has a major advantage : the photon does not self-interact. The inconvenience is that gauge fixing becomes compulsory. We chose the following gauge conditions :

$$
\left\{\begin{aligned}
\forall p_{0} \neq 0, \quad \hat{A}_{0}\left(p_{0}, \mathbf{0}\right) & =0 \\
\forall p, \sum_{k=1}^{3} \widetilde{p}_{k} \hat{A}_{k}(p) & =0
\end{aligned} \quad \text { with } \quad \widetilde{p}_{\mu}=2 \sin \left(\frac{p_{\mu}}{2}\right)\right.
$$

This choice has the practical advantage that it allows to solve explicitely the gauge constraints during field generation ( $c f$. appendix of [10]).

The action $S$ associated with the above Lagrangian is a quadratic form, so generating random fields according to the law $\mathrm{D} A \exp (-S[A])$ to compute the path integral of the theory is simply a multi-dimensional Gaussian random number drawing. This is done in momentum space, where the correlation matrix of the law is quite sparse, and then the position representation of the field is recovered using a Fast Fourier Transform algorithm. The associated U(1) compact field (links) $U_{\mu}^{\mathrm{QED}}$ is then constructed in the following way :

$$
U_{\mu}^{\mathrm{QED}}=\exp \left(i q A_{\mu}\right)
$$

where $q$ is an electric charge.

To check the implementation of the field generation code and investigate volume dependence, we computed semi-analytically the expectation value of the plaquette operator $P_{\mu \nu}$ associated with the field $U_{\mu}^{\mathrm{QED}}$ in an infinite volume lattice to compare it with simulation values. The results are summarized in Figure 1. It appears that for lattice extents greater than 10 the plaquette does not suffer from finite-volume effects and hypercubic invariance is present even with our non symmetric gauge choice (2.7).

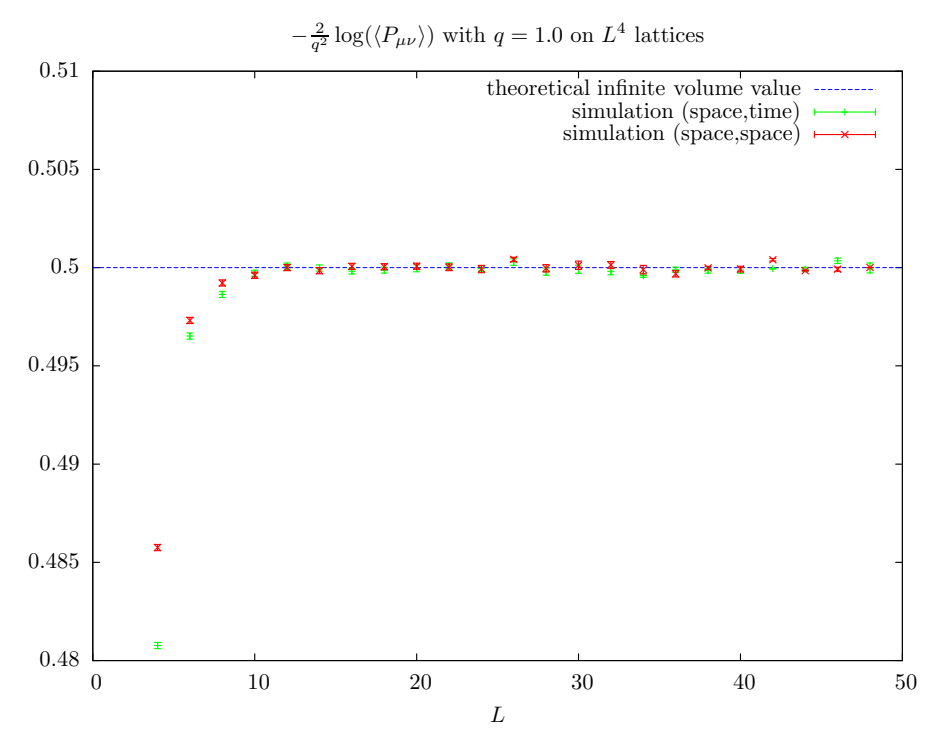

Figure 1: Logarithm of the U(1) plaquette vs. lattice extent $L$. The dashed blue line represents the semianalytical, infinite-volume prediction. The green points correspond to plaquettes aligned along the time and a space directions and the red ones to plaquettes aligned along two perpendicular space directions. 


\section{Coupling to quarks}

Here we describe how to perform a QCD and quenched QED analysis by re-using previously generated SU(3) gauge configuration. We are using $N_{f}=2+1$ QCD simulations with LüscherWeisz gauge action, tree level $\mathrm{O}(a)$-improved Wilson fermions and two steps of HEX smearing (cf. $[12,13]$ for details).

Ideally one would include the $\mathrm{U}(1)$ degrees of freedom directly in the Hybrid Monte-Carlo. But doing this to reproduce the wide range of parameters already explored in BMW collaboration ensembles is extremely expensive. If, in a first instance, one accepts to quench QED, then there is a simple way to re-use previously generated $\mathrm{SU}(3)$ fields. To compute the propagator of a quark $q$ of charge $Q_{q} \in\left\{\frac{2}{3},-\frac{1}{3}\right\}$ proceed as follows :

1. generate an electromagnetic potential $A_{\mu}$ as described in Section 2

2. construct the associated $\mathrm{U}(1)$ field $U_{\mu}^{\mathrm{QED}}=\exp \left(i Q_{q} e A_{\mu}\right)$

3. using a previously generated $\mathrm{SU}(3)$ field $U_{\mu}^{\mathrm{QCD}}$, construct the $\mathrm{U}(3)$ field $U_{\mu}=U_{\mu}^{\mathrm{QED}} U_{\mu}^{\mathrm{QCD}}$

4. compute the quark propagator inverting the Dirac-Wilson operator using $U_{\mu}$ as the gauge field in covariant derivatives

Because Wilson fermions break chiral symmetry explicitly, they require an additive renormalization in the quark masses. This additive renormalization has already been accounted for in our pure QCD simulation. However, in the presence of an extra $U(1)$ gauge interaction, we expect an additional additive renormalization term, that will have to be subtracted, of order $\mathrm{O}\left(Q_{q}^{2} \frac{\alpha}{a}\right)$ where $a$ is the lattice spacing. While suppressed by $\alpha$, this divergence may still be large because it is enhanced by $a^{-1}$. Moreover, this correction breaks the mass isospin symmetry of our $N_{f}=2+1$ simulations, since $Q_{u} \neq Q_{d}$. We have chosen to subtract this divergence by retuning the bare $u$ and $d$ quark masses in such a way that the renormalized masses are equal. Thus, we substract a quantity $Q_{q}^{2} \delta$ from each bare quark mass and tune $\delta$ to a value $\delta_{c}$ where isospin symmetry is recovered. This isospin tuning works very well, as shown in Figure 2.

Everything is now ready to perform a QCD+QED analysis in the mass isospin limit to investigate the violations to Dashen's theorem.

\section{Preliminary results}

Here are our first preliminary results, using the following subset of the BMW collaboration QCD ensembles :

\begin{tabular}{|c|c|c|c|c|c|c|}
\hline$\beta$ & $m_{u d}^{0}$ & $m_{s}^{0}$ & size & $N_{\text {conf }}$ & $\sim M_{\pi}(\mathrm{MeV})$ & $M_{\pi} L$ \\
\hline 3.31 & -0.08500 & -0.04 & $32 \times 16^{3}$ & 218 & 420 & 4.05 \\
3.31 & -0.09300 & -0.04 & $48 \times 24^{3}$ & 128 & 300 & 4.26 \\
3.31 & -0.09530 & -0.04 & $48 \times 24^{3}$ & 210 & 250 & 3.61 \\
3.31 & -0.09756 & -0.04 & $48 \times 32^{3}$ & 130 & 200 & 3.86 \\
\hline
\end{tabular}




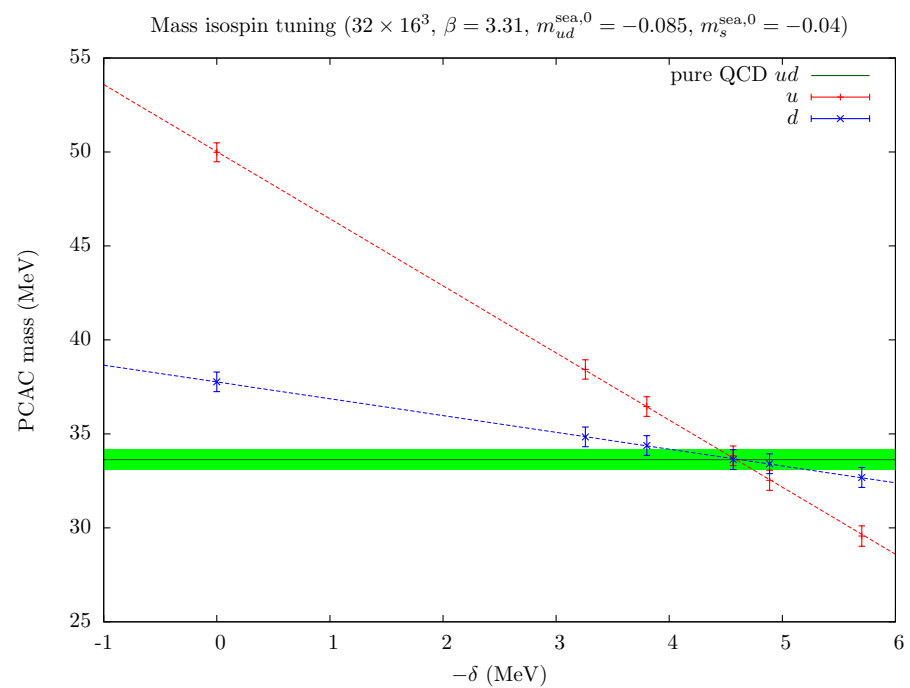

Figure 2: PCAC light quark masses vs. $-\delta$. The behaviour in $\delta$ appears to be perfectly linear. One can sees the large contribution of the $a^{-1}$ additive renormalization at $\delta=0$, where no substraction is applied.

This subset contains a single lattice spacing estimated to be $a \simeq 0.116 \mathrm{fm}\left(a^{-1} \simeq 1.7 \mathrm{GeV}\right)$, one strange quark mass approximatively tuned to its physical value and four pion masses, from $420 \mathrm{MeV}$ down to $200 \mathrm{MeV}$. Clover improvement is done only on SU(3) fields.

Time correlators of $\pi^{0}, \pi^{+}, K^{0}$ and $K^{+}$are constructed by contracting quarks propagators computed as described in Section 3. The $\pi^{0}$ correlator, is constructed by averaging the $u \bar{u}$ and $d \bar{d}$ pseudoscalar propagators, neglecting disconnected contributions. These contributions are subleading in $\alpha$ and $m_{u}-m_{d}$. The mass of a pseudo-Goldstone meson is extracted fitting its time correlator to a hyperbolic cosine. The square of this mass is then extrapolated to the physical $\pi^{+}$ mass $\left(M_{\pi^{+}}^{\phi}=139.57018 \mathrm{MeV}\right)$ using a Taylor expansion around $M_{\pi^{+}}^{\phi 2}$ (as done for [14]) :

$$
M_{P}^{2}\left(M_{\pi^{+}}^{2}\right)=M_{P}^{\phi 2}\left[1+\sum_{k=0}^{n} c_{k}\left(M_{\pi^{+}}^{2}-M_{\pi^{+}}^{\phi 2}\right)^{k}\right]
$$

With this methodology, the previously described QCD ensembles and a linear extrapolation yield the preliminary results (quoted errors are statistical only) :

$$
\begin{aligned}
M_{\pi^{0}} & =134.5 \pm 1.1 \mathrm{MeV} \\
\Delta_{\mathrm{EM}} M_{\pi} & =5.1 \pm 1.1 \mathrm{MeV} \\
\Delta_{\mathrm{EM}} M_{\pi}^{2} & =1380 \pm 50 \mathrm{MeV}^{2} \\
M_{K^{+}} & =501.3 \pm 2.0 \mathrm{MeV} \\
M_{K^{0}} & =499.0 \pm 2.0 \mathrm{MeV} \\
\Delta_{\mathrm{EM}} M_{K} & =2.2 \pm 0.2 \mathrm{MeV} \\
\Delta_{\mathrm{EM}} M_{K}^{2} & =2200 \pm 180 \mathrm{MeV}^{2} \\
\Delta_{A} D & =830 \pm 180 \mathrm{MeV}^{2} \\
\Delta_{R} D & =0.60 \pm 0.14
\end{aligned}
$$




\section{Conclusion}

The preliminary results obtained in Section 4 validate our methodology and clearly encourage us to perform much more detailed calculations (lower quark masses, several lattice spacing, different volumes, etc. ) to gain control over systematic errors and to extend these calculations to other interesting hadronic observables.

\section{Acknowledgments}

Computations were performed using HPC resources from GENCI-[CCRT/IDRIS] (grant 52275) and from FZ Jülich, as well as clusters at Wuppertal and CPT. This work is supported in part by EU grants FP7/2007-2013/ERC $n^{0}$ 208740, MRTN-CT-2006-035482 (FLAVIAnet), OTKA grant AT049652 DFG grant FO 502/2, SFB-TR 55, U.S. Department of Energy Grant $n^{0}$ DE-FG0205ER25681, by CNRS grants GDR $n^{0} 2921$ and PICS $n^{0} 4707$.

\section{References}

[1] K. Nakamura et al. (Particle Data Group), Review of Particle Physics, J. Phys. G 37075021 (2010)

[2] R. Dashen, Chiral SU(3) $\otimes \mathrm{SU}(3)$ as a symmetry of the strong interactions, Phys. Rev. 183(5) 1245-1260 (1969)

[3] J.F. Donoghue, B.R. Holstein and D. Wyler, Electromagnetic self-energies of pseudoscalar mesons and Dashen's theorem, Phys. Rev. D 47(5) 2089-2097 (1993)

[4] J. Bijnens, Violations of Dashen's theorem, Phys. Let. B 306(3-4) 343-349 (1993)

[5] R. Baur and R. Urech, Corrections to Dashen's theorem, Phys. Rev. D 53(11) 6552-6557 (1996)

[6] J. Bijnens and J. Prades, Electromagnetic corrections for pions and kaons: masses and polarizabilities, Nucl. Phys. B 490(1-2) 239-271 (1997)

[7] D.N. Gao, B.A. Li, M.L. Yan, Electromagnetic mass splittings of $\pi, a_{1}, K, K_{1}(1400)$, and $K^{*}(892)$, Phys. Rev. D 56(7) 4115-4132 (1997)

[8] J. Bijnens and N. Danielsson, Electromagnetic corrections in partially quenched chiral perturbation theory, Phys. Rev. D 75(1) 014505 (2007)

[9] A. Duncan, E. Eichten and H. Thacker, Electromagnetic splittings and light quark masses in lattice QCD, Phys. Rev. Let. 76(21) 3894-3897 (1996)

[10] T. Blum et al. (RBC collaboration), Determination of light quark masses from the electromagnetic splitting of pseudoscalar meson masses computed with two flavors of domain wall fermions, Phys. Rev. D 76(11) 114508 (2007)

[11] S. Basak et al. (MILC collaboration), Electromagnetic splittings of hadrons from improved staggered quarks in full QCD, PoS (Lattice 2008) (127) (2008)

[12] S. Dürr et al. (BMW collaboration), Lattice QCD at the physical point: simulation and analysis details, arXiv 1011.2711v1 (2010)

[13] T. Kurth et al. (BMW collaboration), Scaling study for 2 HEX smeared fermions : hadron and quark masses, PoS (Lattice 2010) (2010)

[14] S. Dürr et al. (BMW collaboration), Ab Initio Determination of Light Hadron Masses, Science 322 (2008) 